\title{
X-ray Studies of Flaring Plasma
}

\author{
B. Sylwester ${ }^{1, *}$, J. Sylwester ${ }^{1} \&$ K. J. H. Phillips ${ }^{2}$ \\ ${ }^{1}$ Space Research Center, Polish Academy of Sciences, Kopernika 11, 51-622 Wroclaw, Poland. \\ ${ }^{2}$ Mullard Space Science Laboratory, Holmbury St. Mary, Dorking, RH5 6NT, UK. \\ *e-mail:bs@cbk.pan.wroc.pl
}

\begin{abstract}
We present some methods of X-ray data analysis employed in our laboratory for deducing the physical parameters of flaring plasma. For example, we have used a flare well observed with Polish instrument RESIK aboard Russian CORONAS-F satellite. Based on a careful instrument calibration, the absolute fluxes in a number of individual spectral lines have been obtained. The analysis of these lines allows us to follow the evolution of important thermodynamic parameters characterizing the emitting plasma throughout this flare evolution.
\end{abstract}

Key words. Sun-flares-X-rays—spectroscopic plasma diagnostic.

\section{Introduction}

Often used and a simple method of coronal plasma parameters determination (such as mean temperature and density) is the lines/filters ratio technique. Although an isothermal assumption on which this method relies on is not fully applicable for many solar features, it is frequently used as a first approximation. In the present study, we incorporate also a more advanced analysis approach for a selected flare on 2002 October 4 (max. at 05:38 UT) well observed by Polish spectrometer RESIK. The nominal wavelength coverage of RESIK is $3.35 \AA-6.1 \AA$ in four independent channels. This range contains many emitting lines of $\mathrm{Si}, \mathrm{S}$, Ar, and $\mathrm{K}$ suitable for diagnostics of emitting plasma. A detailed description of the instrument, its operation and calibration is presented in the paper by Sylwester et al. (2005). Our selected event occurred on the solar disc (S18W08) and was of M4.0 class in GOES classification ( $1 \mathrm{~N} \mathrm{in} \mathrm{H} \alpha)$. The complementary data available for this flare are the $3 \mathrm{~s}$ GOES fluxes and the hard X-ray data from RHESSI. The GOES data have been used to determine the temperature and emission measure in the isothermal approximation. We have reconstructed RHESSI images at several times and for different energy bands using the CLEAN algorithm from the SolarSoft package. We assumed a constant volume of emitting region based on the contour profile drawn at $50 \%$ of maximum on RHESSI image (3-6 keV).

\section{The data analysis}

In Fig. 1 we present the four RESIK spectra observed during $50 \mathrm{~s}$ of the rise phase of the analysed flare. The identification of basic lines is given in the papers by 

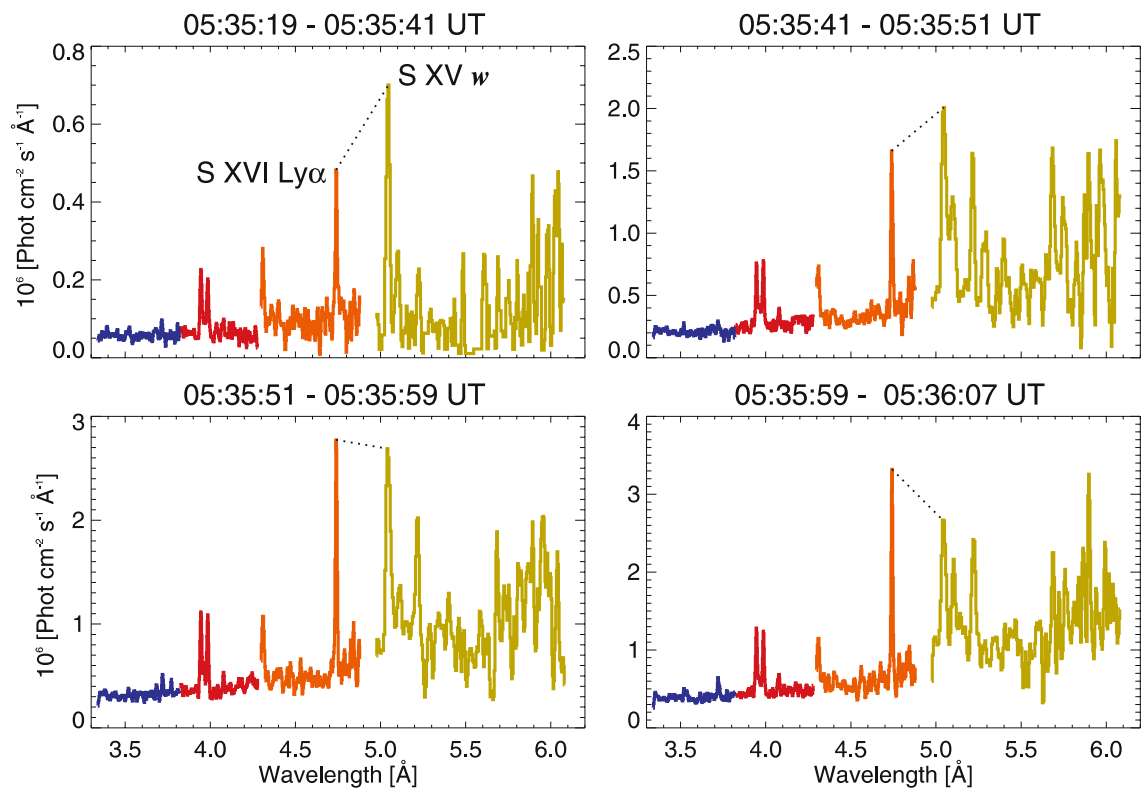

Figure 1. The four RESIK spectra observed during the rise phase of the flare on 2002 October 4 . The data gathering intervals of consecutive spectra are $22,10,8$ and $8 \mathrm{~s}$ respectively. Individual channels are presented in different colours.

Sylwester et al. (2003, 2005) and Kepa et al. (2006). In the figure two lines are labeled and connected with the dotted line in order to show a very fast rate of change in this wavelength band. Denoted lines correspond to the $1 s-2 p$ transitions in $\mathrm{H}$ - and He-like ions of $\mathrm{S}$ (at $4.73 \AA$ and $5.02 \AA$ respectively). The time variability of individual lines and/or bands observed by RESIK may be used as the basis for important plasma parameters determination. We have used the total fluxes (lines and continuum) measured in the most energetically separated RESIK channels ( 1 and 4 ) in order to study the average $T_{e}$ and $E M=N_{e}^{2} V$. These bands cover the $3.4 \AA-3.8 \AA$ and $5.0 \AA-6.05 \AA$ anges respectively. In the left panel of Fig. 2, selected normalized light curves characterizing the different spectral regions are shown. As we have the volume $V$ of the emitting region available (from reconstructed RHESSI images) we can derive the density $N_{e}$

$$
N_{e}=\left(\frac{E M}{V}\right)^{\frac{1}{2}},
$$

and corresponding thermal energy content $E_{t h}$ of the region

$$
E_{t h}=3 k T_{e}(E M / V)^{\frac{1}{2}} .
$$

Here $k$ is the Boltzmann constant.

We have independently performed the corresponding calculations using GOES and RESIK data in two energy bands and the resulting thermal energy content is presented in the right panel of Fig. 2. It is seen that both instruments give very similar quantitative results with the maximum value of thermal energy content $E_{t h}=1.5 \times 10^{30} \mathrm{ergs}$. 

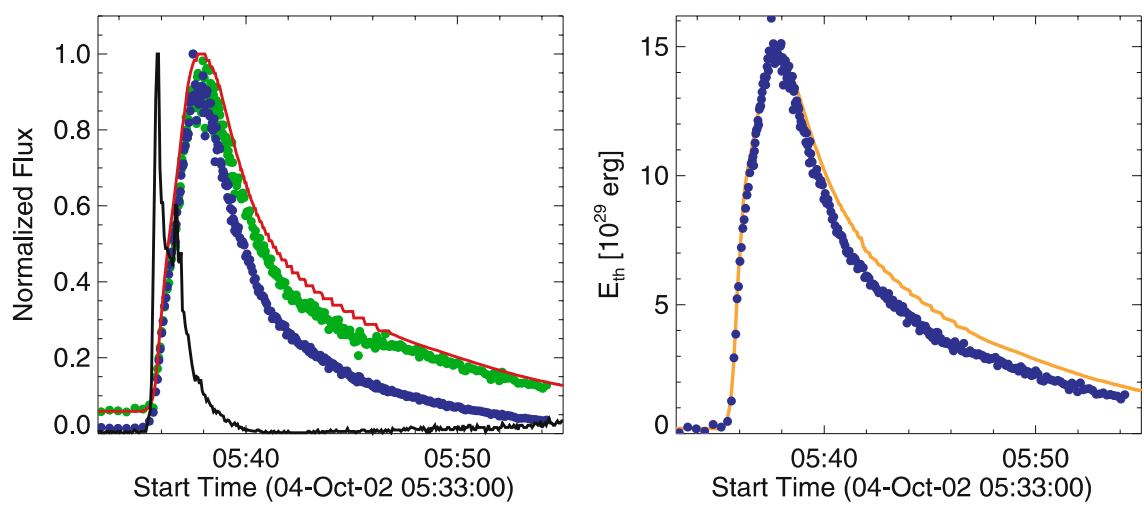

Figure 2. Left panel: The normalized light curves: thin black line corresponds to RHESSI data $(25-50 \mathrm{keV})$, thin red to GOES $1-8 \AA$ and two dotted lines (blue and green) correspond to RESIK channels' 1 and 4 fluxes respectively. Right panel: The thermal energy content of the flaring region as obtained based on the GOES (thin red curve) and RESIK (thick blue dots) data.

Having had the fluxes in many spectral lines/bands observed instantaneously by RESIK we can calculate the momentary distributions of the plasma with the temperature (differential emission measure distributions, DEM) for particular times. In this respect, we have selected 18 narrow spectral bands containing intense lines and continuum from the RESIK spectra. The fluxes measured within these spectral bands have been time-integrated over a sequence of one minute intervals during the rise and the maximum phase of the event studied. Towards the decay longer intervals constituted the input set for the DEM calculations. The Withbroe-Sylwester (Sylwester et al. 1980) iterative DEM reconstruction algorithm has been used. Respective theoretical fluxes have been calculated using the CHIANTI code with the new coronal abundances (Sylwester et al. 2008) and Mazzotta ionization equilibrium adopted (Mazzotta et al. 1998). The uncertainties of DEM have been obtained from 100 Monte Carlo simulations of DEM calculations. The result of DEM calculations for one particular time interval centered at 05:49:05 UT is shown together with the uncertainties in the left panel of Fig. 3. The two-component nature of the distribution can be recognized easily. In the right panel the time evolution of DEM is shown in other representations. The two-component nature of DEM is preserved throughout the flare but the temperature and amount of plasma in each component is changing.

\section{Concluding remarks}

Although the assumption of isothermal approximation is oversimplified, using it with caution allows us to draw some important conclusions about the relative variability of temperature, thermal energy content and of plasma heating/cooling (see Jakimiec et al. 1992) evolution. For various times during flare evolution the DEM calculations indicate the presence of a two-component model: rather cool component (temperatures between $3 \mathrm{MK}$ and $5 \mathrm{MK}$ ) and hot (temperatures between $9 \mathrm{MK}$ and $18 \mathrm{MK}$ ). The relative amount of hot and cool plasma is changing during the evolution. The cool component is rather stable with regard to the amount of plasma and the peak temperature while the 

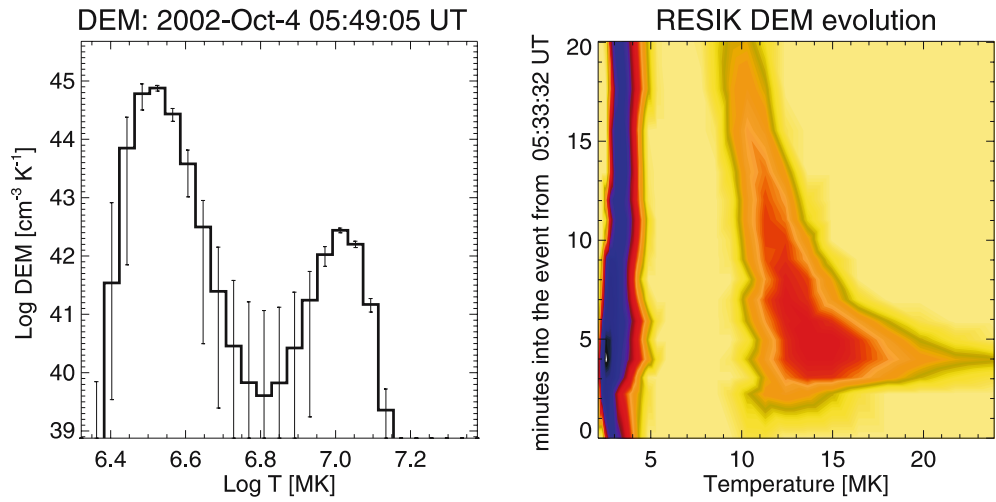

Figure 3. Left panel: The DEM distribution for one particular time with respective error bars indicated. Right panel: The stack (time sequence) of DEM shown in colour shade representation. The time covered (vertical axis) represents the flare evolution from the rise phase (bottom), through the maximum (around minutes 4-6) to the decay phase (top).

properties of hot component are very dynamic. During the cooling phase the maximum temperature of hot plasma component and its amount keep decreasing.

\section{Acknowledgements}

The authors acknowledge support from the Polish Ministry of Education and Science grant 1.P03D.017.29 and a UK Royal Society/Polish Academy of Sciences International Joint Project.

\section{References}

Jakimiec, J., Sylwester, B., Sylwester, J., Serio, S., Peres, G., Reale, F. 1992, Astron. Astrophys., 253, 269.

Kepa, A., Sylwester, J., Sylwester, B., Siarkowski, M., Phillips, K. J. H., Kuznietsov, V. D. 2006, Adv. Space Res., 38, 1534.

Mazzotta, P., Mazzitelli, G., Colafrancesco, S., Vittorio, N. 1998, A\&AS, 133, 403.

Sylwester, J., Schrijver, J., Mewe, R. 1980, Solar Phys., 67, 285.

Sylwester, J., Sylwester, B., Culhane, J. L., Doschek, G. A., Oraevsky, V. N., Phillips, K. J. H. 2003, ESA SP, 535, 733.

Sylwester, J., Gaicki, I., Kordylewski, Z. and 19 other authors 2005, Solar Phys., 226, 45.

Sylwester, J., Sylwester, B., Landi, L., Phillips, K. J. H., Kuznietsov, V. D. 2008, Adv. Space Res., in print. 